\title{
Suppression of low-energy Andreev states by a supercurrent in $\mathrm{YBa}_{2} \mathrm{Cu}_{3} \mathrm{O}_{7-\delta}$
}

\author{
J. Ngai, P. Morales, and J.Y.T. Wei \\ Department of Physics, University of Toronto, 60 St. George St., Toronto, ON M5S1A7, Canada
}

(Dated: November 6, 2018)

\begin{abstract}
We report a coherence-length scale phenomenon related to how the high- $T_{c}$ order parameter (OP) evolves under a directly-applied supercurrent. Scanning tunneling spectroscopy was performed on current-carrying $\mathrm{YBa}_{2} \mathrm{Cu}_{3} \mathrm{O}_{7-\delta}$ thin-film strips at $4.2 \mathrm{~K}$. At current levels well below the theoretical depairing limit, the low-energy Andreev states are suppressed by the supercurrent, while the gap-like structures remain unchanged. We rule out the likelihood of various extrinsic effects, and propose instead a model based on phase fluctuations in the $d$-wave BTK formalism to explain the suppression. Our results suggest that a supercurrent could weaken the local phase coherence while preserving the pairing amplitude. Other possible scenarios which may cause the observed phenomenon are also discussed.

PACS numbers: 74.72.-h, 74.40.+k, 74.45.+c, 74.50.+r
\end{abstract}

\section{INTRODUCTION}

High critical-temperature $\left(T_{c}\right)$ cuprate superconductors are distinguished from conventional $s$-wave superconductors by a predominantly $d$-wave order parameter $(\mathrm{OP})^{1.2}$. The $d$-wave OP symmetry has led to a wealth of unconventional experimental phenomena. One such phenomenon is the formation of low-energy Andreev states bound on non principal-axis surfaces of cuprate superconductors ${ }^{3.4 .5}$. When probed by quasiparticle tunneling, these Andreev states are manifested as zero-bias peaks (ZBCP) in the conductance spectra of high-impedance tunnel junctions. These Andreev states form when time-reversed quasiparticles interfere constructively via the phase sign change of the $d$-wave OP about its nodal $\left(k_{x}= \pm k_{y}\right)$ axes 3.4 . For $s$-wave superconductors, tunneling involves quasiparticle transmission, and is thus only sensitive to the amplitude of the OP. Tunneling into $d$-wave superconductors involves both quasiparticle transmission and Andreev reflection, and is thus sensitive to both the phase and amplitude of the pair wavefunction ${ }^{3,4.6}$.

Recent interest has been focused on how the $d$ wave Andreev bound states, quasiparticle density of states, and pairing symmetry itself could evolve under an applied supercurrent ${ }^{7.8 .9 .10}$. In this paper, we present a scanning tunneling spectroscopy (STS) study of $\mathrm{YBa}_{2} \mathrm{Cu}_{3} \mathrm{O}_{7-\delta}$ (YBCO) thin-film strips carrying directly-applied supercurrents at $4.2 \mathrm{~K}$. Our study uses the supercurrent to perturb the superconducting OP electrodynamically, rather than thermodynamically as was previously done in magnetic field studies 11.12 . High- $T_{c}$ cuprates have characteristically short coherence lengths, which can be $\sim \mathrm{nm}$ in the basal plane. By virtue of its nanoscale junction size, STS can thus provide coherence-length scale information about the high- $T_{c}$ OP when driven by an applied supercurrent. In our experiment, we observed systematic suppression of the phasesensitive spectral features by the supercurrent, while the amplitude-dependent features remain largely unchanged. We discuss and rule out the likelihood of various ex- trinsic effects, and propose instead a model based on current-driven OP phase fluctuations to describe the phenomenon observed.

\section{EXPERIMENTAL SETUP AND RESULTS}

Our experimental setup is illustrated in the left inset of Figure 1 showing a Pt-Ir tip positioned over a YBCO thin-film strip in an STS geometry, with a $\mathrm{Au}$ contact providing the bias voltage $V$ between tip and sample. STS is performed in the usual manner, by measuring the tunneling current $I_{t}$ with the scanning and feedback temporarily suspended, but while a current $I_{s}$ is simultaneously applied through the superconducting film at $4.2 \mathrm{~K}$. In this STS geometry, the high junction impedance $(\sim 0.1 \mathrm{G} \Omega)$ ensures that $I_{t}(\sim 1 \mathrm{nA})$ is decoupled from $I_{s}(\sim 100 \mathrm{~mA})$. The $I_{s}$ is supplied from a floating source below, via Au contacts on the ends of the film strip. A special circuitry is used to synchronize $I_{s}$ with the STS feedback and data acquisition ${ }^{13}$. Short duty-cycle $I_{s}$ pulses $\approx 200 \mu$ s in width are used to prevent sample Joule heating, which could occur at high current levels. DC measurements are also made at lower current levels for comparison, in order to rule out transient electronic effects. For our experiment, this versatile STS technique enabled very low-noise $(<5 \mathrm{pA})$ and highly reproducible tunneling $I$ - $V$ measurements at nanometer length scales.

The YBCO thin-film strips used in our experiment were grown by pulsed laser-ablated deposition on $\mathrm{SrTiO}_{3}$ (STO) substrates. Both $\{110\}$ and $\{001\}$ oriented films were made, the former using a templating technique. The films were typically $50 \mathrm{~nm}$ thick and in a $1 \mathrm{~mm} \times 3 \mathrm{~mm}$ strip-line geometry, aligned along $\langle 100\rangle$ and $[1 \overline{1} 0]$ respectively for the $\{001\}$ and $\{110\}$ films. The film strips showed well-defined $T_{c}$ 's $(\approx 87 \mathrm{~K})$ and sizable transport critical-current densities at $4.2 \mathrm{~K}\left(J_{c} \approx 5 \times 10^{6} \mathrm{~A} / \mathrm{cm}^{2}\right.$, defined by the appearance of $1 \mu \mathrm{V} / \mathrm{mm}$ along the strip). Epitaxiality of the films was confirmed by x-ray diffraction and rocking-curve analysis. Film surface quality was 


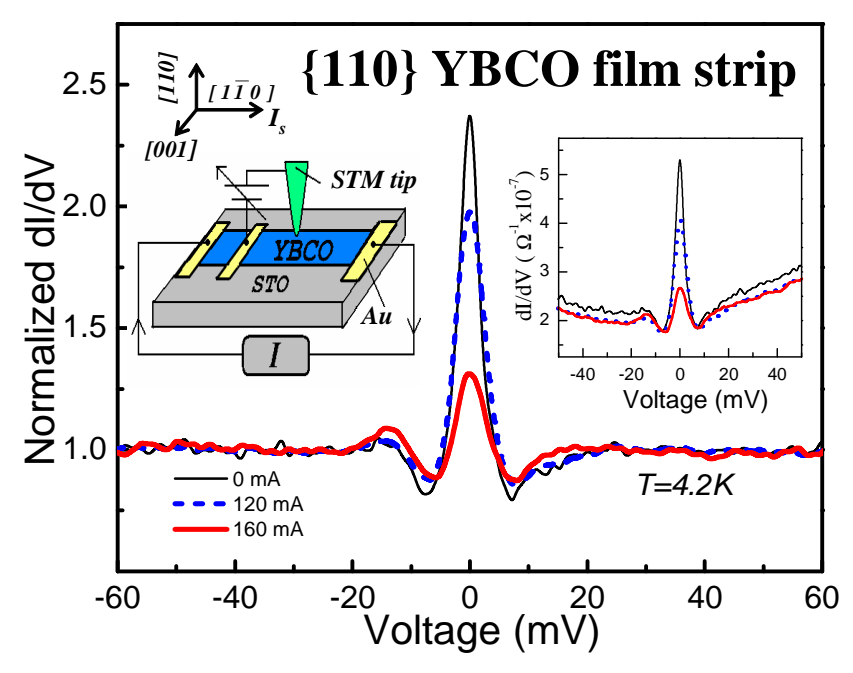

FIG. 1: (Color online) Normalized $d I / d V$ spectra obtained on a $\{110\}$ YBCO film strip carrying a supercurrent $I_{s}$ applied along [110] at $4.2 \mathrm{~K}$. Right inset displays the unnormalized data. Left inset displays our experimental setup showing an STM tip over a YBCO thin-film strip grown on an STO substrate with the orientation as indicated. The tip is biased relative to the sample by a gold pad on the sample surface. Spectroscopy measurements are made while a simultaneous current is passed through the film strip.

determined directly from topography measurements using our STS setup. Shown in the inset of Figure 4 is a topographic image taken on a $\{001\}$ film, indicating smooth terraces with large $c$-axis faces $\sim 30 \mathrm{~nm}$ in size and $a b$-plane edges $\sim 3 \mathrm{~nm}$ in height.

Figure 1 shows tunneling conductance data taken at $4.2 \mathrm{~K}$ on a $\{110\}$ film strip at various current levels applied along [1110]. The $d I / d V$ spectra, which were numerically differentiated from the $I-V$ data, are plotted in the right inset, and the normalized $d I / d V$ spectra are plotted in the main panel. These spectra show a welldeveloped ZBCP structure which is suppressed in height but only slightly changed in width by the applied current. Such ZBCP structures have been commonly observed in YBCO, and could be identified as the low-energy Andreev states based on a predominant $d$-wave OP OP., $^{3.4}$. Two features of the ZBCP spectral evolution versus current are noteworthy. First, the ZBCP shows no splitting, indicating time-reversal invariance of the $d$-wave OP up to $160 \mathrm{~mA}$ (the maximum applied current) in our nearoptimally doped YBCO samples at 4.2K. Second, the area under $d I / d V$ is not conserved, indicating negligible thermal broadening, with a clear loss of Andreev states. The non-splitting of the ZBCP we observed is quantitatively consistent with a recent theoretical calculation ${ }^{7}$, which shows no Doppler shift $\frac{11.12}{1}$ of the Andreev bound states in this geometry until a higher supercurrent density. However, the clear loss of Andreev states we observed was not anticipated by this mean-field calculation.

\section{ANALYSIS}

We have considered various extrinsic effects in analyzing the phenomenon we observed. One possible extrinsic effect is the Joule heating from the sizable current applied. However, our pulsed current technique, along with the absence of significant ZBCP broadening, largely rule out this possibility. A second possible extrinsic effect involves current-generated vortices that could introduce Aharonov-Bohm phases in the Andreev interference process 14 . We note that our current levels are well below the theoretical depairing limit $\underset{7}{ }$, and also below any sharp voltage onsets associated with vortex depinning, although our present experimental resolution cannot yet rule out the presence of such vortices. Nevertheless, the theoretical calculation in Ref.14 has predicted a ZBCP broadening which we do not observe, thus rendering this vortex scenario unlikely. A third possible extrinsic effect involves excess low-energy quasiparticles due to the applied nodal current $\frac{15}{15}$. In this scenario, the excess quasiparticles could effectively raise the quasiparticle temperature, again creating spectral broadening which we do not observe. In summary, all three extrinsic effects described above would be expected to yield a conservation of the low-energy Andreev states, and are therefore unlikely to explain our observations.

Having ruled out the likelihood of these various extrinsic scenarios, we propose instead an explanation based on current-driven OP phase fluctuations to describe the observed phenomenon. High-temperature superconducting cuprates are distinguished by their low carrier densities and short coherence lengths $\frac{16}{}$. These properties imply an inherently low superfluid stiffness, making the order parameter susceptible to fluctuations in its phase $\frac{17}{17}$. Motivated by this possibility, we incorporate phase fluctuations into the generalized BlonderTinkham-Klapwijk (BTK)ํㅗㄹ ${ }^{18}$ formalism, which has been theoretically established ${ }^{4}$ for $d$-wave superconductors, and experimentally verified for $\mathrm{YBCO}^{6}$. The generalized BTK expression for tunneling conductance $G_{n s}$ is given by ${ }^{3,4,6}$ :

$$
\frac{G_{n s}}{G_{n n}}=\int_{-\frac{\pi}{2}}^{+\frac{\pi}{2}} d \theta \int_{-\infty}^{+\infty} d E\left[1+|a|^{2}-|b|^{2}\right] \frac{\partial f(E-e V)}{\partial V}
$$

where $a$ and $b$ are the Andreev-reflection and normalreflection coefficients, $f$ is the Fermi-Dirac function, $G_{n n}$ is the normal-state junction conductance, $E$ the quasiparticle energy, and $\theta$ the polar angle in $k$-space. To model the effects of OP phase fluctuations, we modify the BTK kernel from Ref. 4 by adding a phase factor $\mathrm{e}^{i \varphi}$ to the phase-interference term in its denominator (see Eqn 2below) then integrating it over the domain of phase 


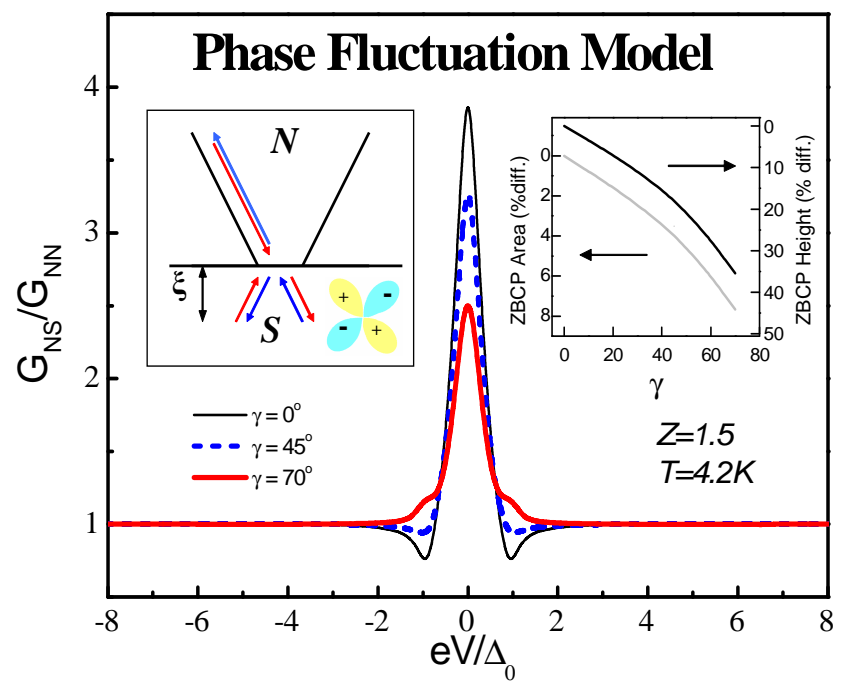

FIG. 2: (Color online) Phase fluctuation model showing the evolution of the ZBCP on a $\{110\}$ junction as a function of the phase smearing parameter $\gamma$. Left inset illustrates the Andreev interference process based on the $d$-wave phase sign change. Right inset shows the ZBCP height and spectral area as a function of $\gamma$.

$\varphi$ weighted by a Gaussian of width $\gamma$ :

$$
\begin{gathered}
1+|a|^{2}-|b|^{2}=\int_{-\pi}^{+\pi} d \varphi \frac{1}{\sqrt{2 \pi \gamma}} e^{-\varphi^{2} / \gamma^{2}} \times \\
\frac{16\left(1+\left|\Gamma_{+}\right|^{2}\right) \cos ^{4} \theta+4 Z^{2}\left(1-\left|\Gamma_{+} \Gamma_{-}\right|^{2}\right) \cos ^{2} \theta}{\left|4 \cos ^{2} \theta+Z^{2}\left[1-\Gamma_{+} \Gamma_{-} e^{i \phi_{-}-i \phi_{+}+i \varphi}\right]\right|^{2}}
\end{gathered}
$$

Note that the amplitude factors $\Gamma_{ \pm}=\left(E /\left|\Delta_{ \pm}\right|\right)-$ $\sqrt{\left(E /\left|\Delta_{ \pm}\right|\right)^{2}-1}$ contain a $d$-wave gap function with $\Delta_{0}$ as gap maximum. The phase factors $e^{i \phi_{ \pm}}=\Delta_{ \pm} /\left|\Delta_{ \pm}\right|$ represent the sign of the pair potential $\Delta_{ \pm}=\Delta\left(\theta_{ \pm}\right)$ which is experienced by an Andreev-reflected quasielectron (or quasihole) propagating at an angle $\theta_{+}$(or $\theta_{-}=$ $\pi-\theta_{+}$) relative to the junction normal (see left inset in Fig.3). Through the extra phase introduced by $e^{i \varphi}$, the weighted integral over $\varphi$ essentially smears out the relative phase between consecutive Andreev-reflected quasiparticles. This phase smearing would cause incoming and outgoing quasiparticles to interfere less constructively, thereby leading to a loss of the low-energy Andreev states. Our phenomenological approach can be compared with the more rigorous treatment of Ref.19 which uses a 2D X-Y model based on vortex fluctuations ${ }^{20}$. Our model makes no assumptions about which physical mechanism is actually driving the phase fluctuations, while also going beyond the low-impedance $(Z \ll 1)$ and principle-axis $(\{100\})$ junction cases $\frac{19}{}$.

Our spectral simulations for a $\{110\}$ junction are plotted in Figure 2] as normalized conductance $G_{n s} / G_{n n}$. A large barrier parameter $Z=1.5$ was used to represent our high-impedance junctions, along with the experimental temperature $T=4.2 \mathrm{~K}$ and a typical gap maximum $\Delta_{0}=15 \mathrm{meV}$. A clear trend of ZBCP suppression is seen

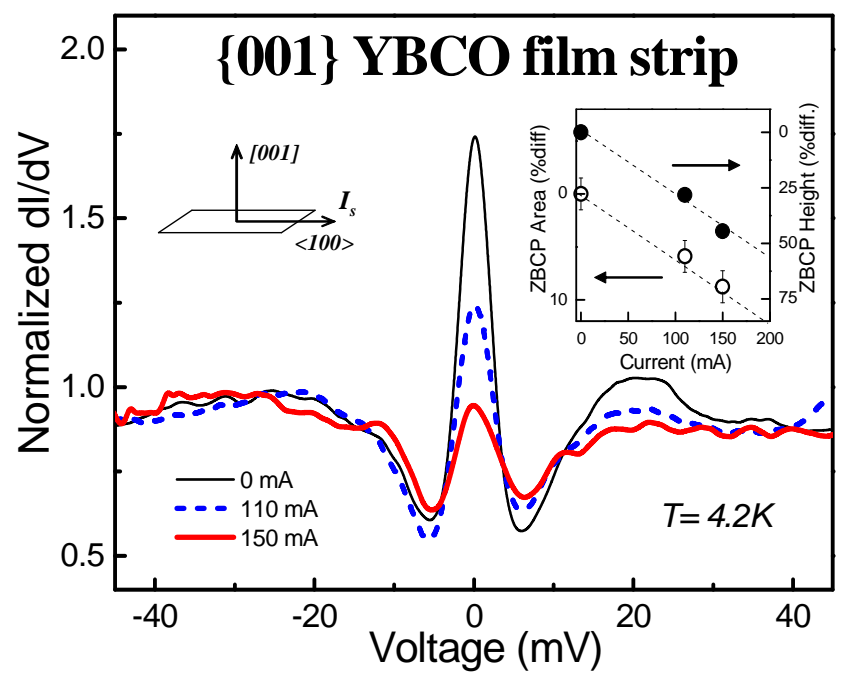

FIG. 3: (Color online) Normalized $d I / d V$ spectra obtained on a $\{001\}$ YBCO thin-film strip carrying a supercurrent $I_{s}$ applied predominantly along $<100>$ at $4.2 \mathrm{~K}$. Right inset shows the evolution of the ZBCP height and spectral area as a function of the applied current.

versus the phase-smearing parameter $\gamma$, showing a drop in the peak height and a loss of spectral area. Detailed evolutions of height and area versus $\gamma$ are plotted in the right inset of Fig 2. Comparing our data in Fig 1 with the simulations in Figure 22 it is clear that the ZBCP suppression could be qualitatively attributed to the dephasing effects of current-driven OP phase fluctuations. Physically speaking, consecutive Andreev-reflected quasiparticles which are bound within a coherence length $\xi$ from the specular $\{110\}$ surface (see left inset of Fig.2), would experience an effective smearing in their relative phase as a result of the phase fluctuations, thus interfering less constructively. This phase smearing leads directly to a loss in the low-energy Andreev states, resulting in the ZBCP suppression seen in the data.

\section{DISCUSSION}

It is important to consider possible physical mechanisms by which the supercurrent is driving the local phase fluctuations. One scenario involves the possibility of spatial doping inhomogeneities in the superconducting cuprates 21 . In the presence of current flow, local areas of strong superconductivity could become decoupled from each other, thus permitting local phase fluctuations. Another scenario may involve the proliferation of thermally-generated vortex-antivortex pairs in the Kosterlitz-Thouless picture 22.23 , but with current as an additional driving force ${ }^{24,25}$. In principle, the static phase gradient carried by the supercurrent could also couple dynamically with other types of phase-ordered states, such as spin/charge-density waves ${ }^{26.27}$, staggered flux-like states ${ }^{28.29 .30}$ or collective modes ${ }^{31.32}$, to produce 


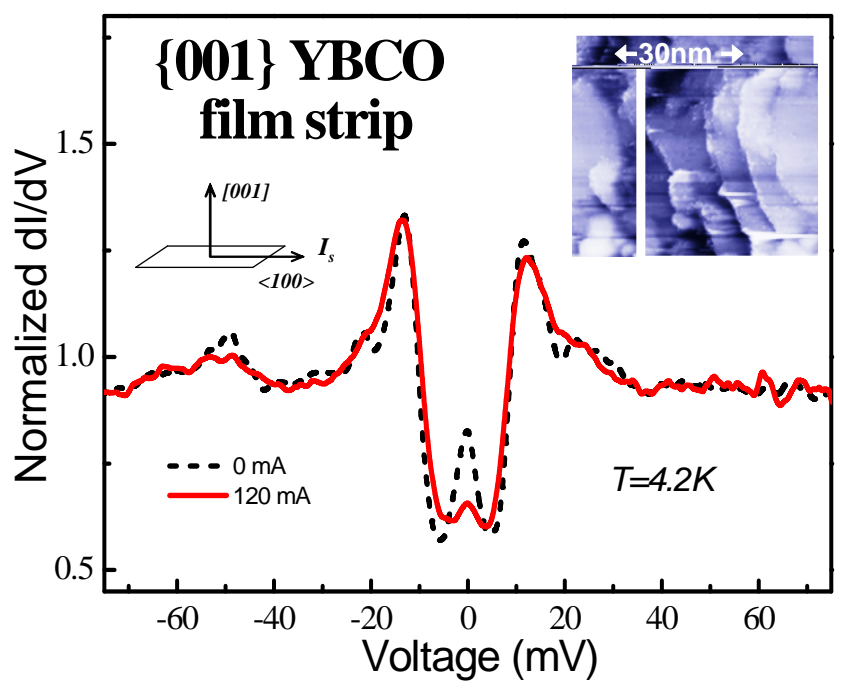

FIG. 4: (Color online) Normalized $d I / d V$ spectra obtained on a $\{001\}$ YBCO thin-film strip carrying a supercurrent $I_{s}$ applied predominantly along $<100>$ at $4.2 \mathrm{~K}$. The inset shows film topography.

enhanced phase fluctuations. In general, any of the above scenarios may lead to local regions containing a weakened OP, with respect to phase fluctuations. In this connection, it is worth noting that such weakened regions could also be nucleation centers for phase-slip phenomena, which were seen in recent transport studies on superconducting $\mathrm{YBCO}$ microstrips 33.34 .

It should also be remarked that our discussion of lowenergy Andreev states is not limited to only specular cases. These Andreev states could also form under nonspecular conditions in a $d$-wave superconductor, i.e. near grain boundaries, extended non-magnetic impurities, or surface interstitial defects ${ }^{35.36 .37 .38 .39 .40 .41}$. Like the specular Andreev bound states, these non-specular resonance states are essentially also based on $d$-wave Andreev interference, and should therefore also be conserved under an applied current, as was shown in a recent theoretical calculation ${ }^{42}$. However, another calculation for this nonspecular case, done as a function of an applied magnetic field, indicated a non-conservation of Andreev states 43 . Further theoretical work would be necessary to clarify this issue. Experimentally, the technique presented in our present work, measuring the evolution of the Andreev states under a supercurrent, may in fact provide a definitive way to distinguish between the specular and non-specular cases ${ }^{7.42}$.

Finally, we note that ZBCP were also seen on $\{001\}$ samples, showing the same suppression effect by currents applied predominantly along $<100>$. Figure 3 plots an example of such ZBCP at $4.2 \mathrm{~K}$. Such ZBCP have previously been observed on $c$-axis cuprate thin films 44 , and could be explained by the presence of terrace edges on the film surface (see inset of Fig (4) which would allow in-plane tunneling to occur on nominally $c$-axis samples. The probability for in-plane tunneling could also be enhanced by the inherently larger matrix element for in-plane versus $c$-axis tunneling 46 . Consistent with this picture are instances where the ZBCP is seen together with a gap structure at the same tip location on $\{001\}$ samples. The main panel in Fig 4 shows such a spectrum, with a ZBCP flanked by well-developed gap edges at $\approx \pm 14 \mathrm{meV}$. The gap structures could be identified as the $d$-wave averaged density of states associated with $c$-axis tunneling 6.45 .46 . Since $c$-axis tunneling does not involve Andreev interference, the gap structures are expected to depend only on the OP amplitude and be relatively insensitive to the OP phase fluctuations. What we observe in Fig 4 is entirely consistent with this expectation. Upon the application of a supercurrent, the ZBCP is suppressed as before but without significant variation in the gap structure. This is a remarkable observation, indicating that an applied supercurrent could weaken the local phase coherence while maintaining the pairing amplitude.

\section{CONCLUSION}

In summary, using a scanning tunneling spectroscopy technique we have observed nanoscale suppression of lowenergy Andreev states by a directly-applied supercurrent in $\mathrm{YBa}_{2} \mathrm{Cu}_{3} \mathrm{O}_{7-\delta}$ thin-film strips at $4.2 \mathrm{~K}$. The distinct non-conservation of Andreev states indicates that this phenomenon cannot be easily attributed to extrinsic effects such as Joule heating, vortex flow or excess quasiparticles. We have analyzed this suppression in terms of order-parameter phase fluctuations, using a model based on the $d$-wave BTK formalism. Qualitative agreement between our model and data suggests that the supercurrent may induce local phase fluctuations in the hightemperature superconducting cuprates. Further work is needed to elucidate this nanoscale phenomenon.

\section{Acknowledgments}

This work was supported by grants from NSERC, CFI, OIT, MMO/EMK, and the Canadian Institute for Advanced Research in the Quantum Materials Program.
1 C.C. Tsuei and J.R. Kirtley, Rev. Mod. Phys. 72, 969 (2000); C.C. Tsuei, J.R. Kirtley, G. Hammerl, J. Mannhart, H. Raffy, and Z.Z. Li, Phys. Rev. Lett. 93,
187004 (2004).

2 D.J. Van Harlingen, Rev. Mod. Phys. 67, 515 (1995).

${ }^{3}$ C.-R. Hu, Phys. Rev. Lett. 72, 1526 (1994). 
4 Y. Tanaka and S. Kashiwaya, Phys. Rev. Lett. 74, 3451 (1995).

5 For reviews, see: S. Kashiwaya and Y. Tanaka, Rep. Prog. Phys. 63, 1641 (2000); Löfwander, V.S. Shumeiko and G. Wendin, Supercond. Sci. Technol. 14, R53-R77, (2001); G. Deutscher, Rev. Mod. Phys. 77, 109 (2005).

6 J.Y.T. Wei, N.-C. Yeh, D.F. Garrigus, and M. Strasik, Phys. Rev. Lett. 81, 2542 (1998).

7 D. Zhang, C. S. Ting, and C.-R. Hu, Phys. Rev. B 70, 172508 (2004).

8 I. Khavkine, H.-Y. Kee, and K. Maki, Phys. Rev. B 70, 184521 (2004).

9 M. Zapotocky, D. L. Maslov, P. M. Goldbart, Phys. Rev. B 55, 6599 (1997)

${ }^{10}$ V. V. Kabanov, Phys. Rev. B, 69, 052503 (2004).

11 M. Aprili, E. Badica, and L.H. Greene, Phys. Rev. Lett. 83, 4630 (1999), and references therein.

12 R. Krupke and G. Deutscher, Phys. Rev. Lett. 83, 4634 (1999).

13 J. Ngai, Y.C. Tseng, P. Morales, V. Pribiag, J.Y.T. Wei, F. Chen, and D.D. Perovic, Appl. Phys. Lett. 84, 1907 (2004).

14 Y. Asano, Y. Tanaka, and S. Kashiwaya, Phys. Rev. B 69 134501 (2004).

15 D. Xu, S.K. Yip, and J.A. Sauls, Phys. Rev. B 51, 16233 (1995).

16 V. J. Emery and S. A. Kivelson, Nature 374, 434 (1995).

17 L. Merchant, J. Ostrick, R.P. Barber, Jr., and R.C. Dynes Phys. Rev. B 63, 134508 (2001).

18 G.E. Blonder, M. Tinkham, T. M. Klapwijk, Phys. Rev. B, 25, 4515, (1982).

19 H.-Y. Choi, Y. Bang and D.K. Campbell, Phys. Rev. B 61, 9748 (2000).

20 M. Franz and A. J. Millis, Phys. Rev. B 58, 14572 (1998).

21 K.M. Lang, V. Madhavan, J.E. Hoffman, E.W. Hudson, H. Eisaki, S. Uchida, and J.C. Davis, Nature 415, 412 (2002).

22 P. Minnhagen, Rev. Mod. Phys. 59, 1001 (1987).

23 Z. Tesanovic, O. Vafek, and M. Franz, Phys. Rev. B 65, 180511(R) (2002).

24 M. R. Beasley, J. E. Mooij, and T. P. Orlando, Phys. Rev. Lett. 42, 1165 (1979).

${ }^{25}$ K. Epstein, A.M. Goldman and A.M. Kadin, Phys. Rev.
Lett. 47, 534 (1981).

26 E. Demler, S. Sachdev, and Y. Zhang, Phys. Rev. Lett. 87, 067202 (2001).

27 H. L. Edwards, A. L. Barr, J. T. Markert, and A. L. de Lozanne Phys. Rev. Lett. 73, 1154 (1994).

28 J. B. Marston and I. Affleck, Phys. Rev. B 39, 11538 (1989)

29 C. M. Varma, Phys. Rev. B 55, 14554 (1997).

30 S. Chakravarty, R.B. Laughlin, D.K. Morr, and C. Nayak, Phys. Rev. B 63, 094503 (2001).

31 Y. B. Kim and X. G. Wen, Phys. Rev. B 48, 6319 (1993).

32 P. Devillard, R. Guyon, T. Martin, I. Safi, and B.K. Chakraverty, Phys. Rev. B 66, 165413 (2002).

33 F. S. Jelila, J.-P. Maneval, F.-R. Ladan, F. Chibane, A. Marie-de-Ficquelmont, L. Mechin, J.-C. Villegier, M. Aprili, and J. Lesueur, Phys. Rev. Lett. 81, 1933 (1998).

34 P. Morales, M. DiCiano and J. Y.T. Wei, Appl. Phys. Lett. 86, 192509 (2005); Phys. Rev. Lett. (submitted).

35 A. V. Balatsky, M. I. Salkola, and A. Rosengren, Phys. Rev. B 51, 15547 (1995).

36 Y. Tanuma, Y. Tanaka, M. Yamashiro, and S. Kashiwaya, Phys. Rev. B 57, 7997 (1998).

37 I. Adagideli, P.M. Goldbart, A. Shnirman, and A. Yazdani, Phys. Rev. Lett. 83, 5571 (1999).

38 J. X. Zhu, T.K. Lee, C.S. Ting, and C.-R. Hu, Phys. Rev. B 61, 8667 (2000).

39 K. V. Samokhin and M. B. Walker, Phys. Rev. B 64, 172506 (2001).

40 M.S. Kalenkov, M. Fogelström and Y.S. Barash, Phys. Rev. B, 70, 184505 (2004).

41 S.H. Pan, E.W. Hudson, K.M. Lang, H. Eisaki, S. Uchida, and J.C. Davis, Nature (London) 403, 746 (2000).

42 D. Zhang, C. S. Ting, and C.-R. Hu, Phys. Rev. B 71, 064521 (2005).

43 K.V. Samokhin, Phys. Rev. B 68, 104509 (2003).

44 S. Misra, S. Oh, D.J. Hornbaker, T. DiLuccio, J.N. Eckstein, A. Yazdani, Phys. Rev. B 66, 100510(R) (2002).

45 I. Maggio-Aprile, Ch. Renner, A. Erb, E. Walker, and O. Fischer Phys. Rev. Lett. 75, 2754 (1995).

46 J.Y.T. Wei, C.C. Tsuei, P.J.M. van Bentum, Q. Xiong, C.W. Chu, and M.K. Wu, Phys. Rev. B 57, 3650 (1998). 